\title{
Applying a "Hard Science" Disciplined Approach to Identify and Verify Chemical Properties for Safe Response, Handling, and Disposal
}

\author{
Clifford L. Holland \\ Spill Management Inc., Stoney Creek, Canada \\ Email: cliffholland@spillmanagement.ca
}

Received 18 January 2016; accepted 23 February 2016; published 26 February 2016

Copyright (C) 2016 by author and Scientific Research Publishing Inc.

This work is licensed under the Creative Commons Attribution International License (CC BY).

http://creativecommons.org/licenses/by/4.0/

(c) (i) Open Access

\begin{abstract}
Personnel who may have to deal with the uncertain nature and properties of unknown chemicals at an environmental incident, hazardous waste site, industry or in a laboratory need a way to identify the actual risks and hazards they may face. This applies whether the personnel are emergency responders, forensic researchers or waste clean-up crews. There is, however, a quick-check system to identify chemical properties of labeled, unlabeled, unknown or mixed chemicals. The system uses pH paper, starch paper and a source of flame such as a barbeque lighter or Bunsen burner. The basic test takes less than 60 seconds and will identify or verify high-risk properties such as whether substances are oxidizers or reactive to heat, air or water, their range of flammability and their corrosiveness. The test results provide vital information for responders to make critical decisions, whether dealing with a laboratory spill or a full-scale hazardous material emergency. Using hard science helps in dealing with time-critical issues, such as when, or whether, to add water to modify a chemical reaction when a bad situation could turn into a disaster. Whether working with laboratory or industrial chemicals or responding to a major emergency, hard science can provide the information needed to approach the scene, secure the area, and keep unprotected and unauthorized personnel out. It identifies the physical and chemical properties of known, unknown and newly formed substances and allows personnel to assess the risk, hazards and potential scales of impact including when it is safe to respond or take other appropriate action.
\end{abstract}

\section{Keywords}

Response, Chemicals, Safety, Testing, Properties, Emergency 


\section{Introduction}

Safe Response, Handling, and Disposal of chemical at an environmental incident, hazardous waste site, industry or in a laboratory require the verification of the chemicals properties. The spontaneous reactions from mixing of incompatible hazardous wastes or chemical spills in laboratories can hold many unknown and unexpected results for onsite responders or emergency crews and even forensic investigators. For instance, personnel may be uncertain about putting water on a chemical spill because the water may escalate the situation to generate heat, rapid burning or even a detonation. Delays in determining an appropriate response may have serious consequences for safety of first responders and infrastructures. The rapid decomposition of a material can generate a shock wave that may travel outward faster than the speed of sound with enough energy to cause death and damage structures and the environment if personnel cannot check chemical properties and aren't prepared for surprises. Because precautions are not always taken, responders, spill recovery and site remediation experts including investigators, as well as, long term sampling and monitoring personnel who may need to enter the site to collect evidence or perform evaluations or studies, should consider performing some of these tests for personal safety in suitable safe-areas for conducting open flame testing. Plainly put, what chemists, responders and emergency personnel don't know about the properties of chemicals may kill them.

By determining chemical properties and potential reactions, responders can also identify the type of personal protective equipment (PPE) they need, whether goggles and a pair of chemical-proof gloves or a fully encapsulating suit with supplied air. The contamination, which has collected on clothing, vehicles and equipment, will also show up in swab tests.

The disciplined approach relies on a set of First Responder's Golden Rules for approaching a scene: Never assume anything! Suit up to protect entry routes to the body from toxic substances; Work clean, to prevent cross contamination and adverse reactions that can result. The disciplined approach revolves around ASIA-R:

- Approach the scene;

- Secure the scene to keep out unprotected and unnecessary personnel;

- Identify chemical properties;

- Assess risks and hazards;

- Respond or take appropriate action to modify conditions and test, verify and assess danger factors.

This approach means that responders do not enter to patch, repair or open containers to transfer or remove any known or unknown material unless all site safety, proper protective equipment and support services are in place.

The first personnel on the scene of an incident involving chemicals must be aware of all the possible risks, hazards and dangers including the effects of toxic, corrosive, flammable and radioactive substances. They need to factor in the possibility of human error and the probability of unplanned events such as a situation that should be routine escalating to a level that threatens health, property and the environment. For instance, lighter-than-air gases can accumulate in a ceiling area, where gas-burning heaters can become an ignition source. Gases with a heavier specific gravity may travel to a sump area where turning on a light could be an ignition source. Entering an area or leaving a door open may also allow flammable vapours to spread, creating conditions for an unexpected emergency.

Spill Management Inc. has been using hard-science to test and verify the properties of chemicals including unknown substances since the 1970s. The biggest concern about dealing with both unknown and known laboratory chemicals was accurately determining chemical properties for safe handling and transport for disposal or treatment at waste treatment facilities. Testing and verifying the physical and chemical properties of known and unknown chemicals, reagents and substances to determine the actual risks, hazards and properties of known and unknown substances became extremely important as concerns for site and personnel safety grew.

It was not uncommon in the 1980s to be involved at digs on the grounds of institutions, which had buried waste chemicals on site because they were too dangerous to dispose of in the garbage. It was vital to everyone's safety to determine if the contents of containers were stable, since high-risk and unstable chemicals could be heat, friction, shock or sunlight sensitive. A $500 \mathrm{ml}$ bottle of some substances could go off with the force of a stick of dynamite.

Based on the activities of facilities and the types of chemicals involved, as well as the blending of small amounts of waste laboratory chemicals, a 170-litre (45-gallon drum) could contain many different waste residues designated for final disposal. The need for a formal testing process became obvious within the waste management industry after first-hand experience with unexpected, violent and explosive reactions whether on spill 
sites, hazardous waste storage sites or when preparing chemicals for disposal. The right test process would also avoid losing time while taking samples and sending them for laboratory analysis and waiting for results. And it would take into account the working conditions, the integrity and types of containers, temperatures of surroundings and the substances.

This paper does not follow a strictly science format that could be expected if the work was experimental. Rather, it deals with the background that led to development of the Process and how it uses hard-science to determine the real properties of chemicals, reagents and substances, without even knowing their chemical names.

\section{Developing a Complete Approach for Safety}

Personnel who enter a hazardous area where an unknown, unwanted or unplanned upset or reaction has occurred should be trained, experienced and knowledgeable about the industry, the products or the processes. They should suit up with proper PPE to cope with a worst-case situation, particularly if they are entering a confined space or other hazardous area. They must be able to identify and determine the risks, hazards, potential scales of impact and the potential for more occurrences. It is also advisable for each responder to carry a flammability monitor or general alarm sniffer. For instance, gases may be generated which can present a health threat, whether immediate or in the long-term, as well as react spontaneously with other substances, whether known or newly-formed substances, to create heat or fire.

A laboratory situation may seem stable because the incompatible substances appear to have already reacted. But the substances might still be sensitive to heat, friction and shock. By not intimately understanding the physical and chemical properties of known, unknown and newly formed substances, personnel may not have any warning of danger until there is another set of reactions, off-gassing, smoldering, heat, fire or an explosion. Personnel simply walking through an area and tracking incompatibles can set off these adverse reactions.

During the 1980s, physical testing of known and unknown chemical properties was effective and being done as needed for personal and onsite safety but was crude by today's standards. In the beginning simple testing involved putting a few drops of a liquid on the end of a paper towel and trying to ignite the substance to determine the risk of combustion, or rapid burning by observation. Soon, $\mathrm{pH}$ paper replaced the paper towel and was able to provide six chemical property determinations. It allowed us to determine if we were dealing with acids, bases or neutral substances. And the paper could be lit to determine the flashpoints of a substance, whether it was non-flammable, combustible or highly flammable. Concern about oxidizers that could react with anything that burns to cause heat, fire and/or explosions prompted the use of potassium iodide paper which allowed further testing for the properties of mixed and blended chemicals and multi-layers of the substances by obtaining multilayer samples with a pipette. By 1989, Spill Management had formalized the Chemical Property Testing Process, based on the results and observations from working daily in the field with unlabeled, mislabeled and unknown solid and liquid substances. The development of the Process also relied heavily on established definitions of chemical properties from publications such as chemical dictionaries [1] [2].

\section{Test Methods}

In the tests to determine and verify chemical properties, small representative samples of substances (liquid, solid or gases determined by using a flammability detector) such as one or two drops are applied to the test papers. The test will determine if a sample is a reducing agent or oxidizer as well as provide corrosive readings for acid, base or neutral substances. With a clear-flame ignition source, substances with known or unknown properties can be tested for being heat-sensitive, flammable or non-flammable, as well as whether they have high or low flash points to assess the risk of combustion.

The test papers are used in swab tests, dampening the $\mathrm{pH}$ paper with water as necessary, to pick up trace amounts of contamination and identify and verify the stability of a spilled product or mixture of products. These tests will help establish the safe conditions for entry and exit from the scene and the level of contamination on clothing, equipment and vehicles.

All testing should also be done away from hazardous areas and in a safe location, with safety measures that may include a fire extinguisher, a small pail of water and inert sorbent material, depending on the products and circumstances. Ideally, testing will be done outdoors or inside a fume hood.

It can also be wise to test known liquid chemicals and solutions as well as solid chemicals and substances to verify information and to determine factors vital to site safety which may be missing from emergency response 
guide books, material safety data sheets and container labels. There have been many instances over the years where testing has prevented injury and damage or established that conditions were safe for rapid and effective site entry and response. There are also many events where proper procedures for testing substances could have avoided serious situations, loss of critical response time and reduced the scales of impact at major incidents.

\subsection{Testing and Verifying Chemical and Physical Properties of Known and Unknown Solids, Liquids and Gases}

Duotest $\mathrm{pH}$ paper is highly recommended for the tests, because of its versatility. The dual coloured $\mathrm{pH}$ paper with a centre stain indicator may provide more accurate readings for handling, storage and processing chemicals.

The $\mathrm{pH}$ paper should be protected from contaminate or corrosive atmospheres by being stored inside a sealed container when on spill sites. No more than $1.5 \mathrm{~cm}$ (1/2 inch) of product is needed on the end of the test paper for safety in testing many substances. It also means that only small representative samples of material are needed for the tests. When working with the off-gassing of corrosive vapours or other substances, it is beneficial to wet half the length of the test papers with water to identify any airborne properties, which will react more readily with the wet or dry portion of the test paper. Figure 1 summarizes the three steps used within the Quick Check Chemical Testing process and further details for each step are as follows:

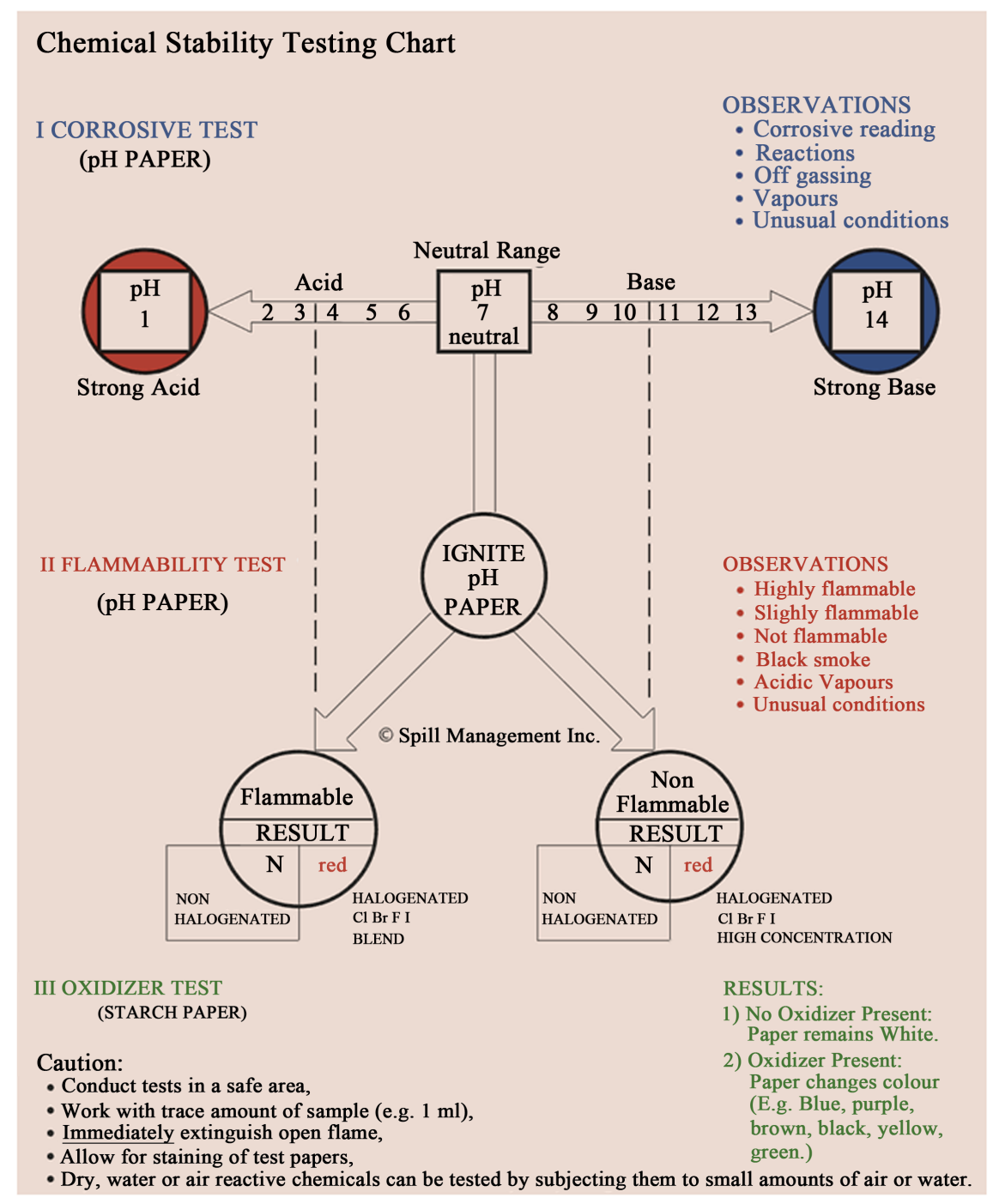

Figure 1. Stability test chart summarizing the three steps used within the quick check chemical testing process. 
Test \#1 Testing for corrosives with $\mathrm{pH}$ paper

For liquid samples dip $1.5 \mathrm{~cm}$ (about 1/2 inch) of $\mathrm{pH}$ paper into liquid sample solution and then match the colour of the $\mathrm{pH}$ paper to the $\mathrm{pH}$ colour indicated on the chart on the dispenser, record the reading and take the same test paper to Test No. 2. When materials to be tested are solid, wet $1.5 \mathrm{~cm}$ of test paper with water and touch paper to substance and compare paper to chart. Note: Some samples may generate corrosive off-gassing, which will cause the $\mathrm{pH}$ paper to immediately change colour before the $\mathrm{pH}$ paper even comes in direct contact with the sample to be tested. For gaseous sampling wet $1.5 \mathrm{~cm}$ of test paper and place in gaseous atmosphere for an immediate result and then compare paper to indicator chart. This early reading would be a warning to site personnel.

Test \#2 A two-part test for flammability

When completing this test it is important to have fire suppression equipment on hand, even if it is just a beaker of water. It is also wise to use a longneck butane barbeque lighter as the ignition source. Produce a flame using an ignition source and pass the $\mathrm{pH}$ paper sample from Test \#1 through the flame quickly. If it ignites, blow the flame out immediately and record the results as highly flammable. If the test paper did not ignite, hold the paper in the flame for two seconds. If the substance was slow to ignite, record the results as combustible. If the paper does not ignite, record the results as non-flammable.

Test \#3 Using potassium iodide starch paper to test for oxidizers

For liquid samples dip $1.5 \mathrm{~cm}$ of starch paper into the liquid, look for a change of colour or creation of a fine line. When materials to be tested are solid wet $1.5 \mathrm{~cm}$ and rub the solid substance, looking for a change of colour or a fine line. For gaseous sampling, wet $1.5 \mathrm{~cm}$ of starch paper and expose the paper to the gaseous atmosphere, looking for an immediate colour change or a fine line. If there is any change of colour from the original white or a fine line appears, treat the substance as an oxidizer. If the starch paper remains white, treat the substance as a reducing agent or oxygen scavenger.

\subsection{Identification of Laboratory and Industrial Waste Chemicals Using the Quick Check Chemical Testing Process}

Figure 2 summarizes, in picture format, the results of the analysis of samples of typical laboratory and industrial waste chemicals. Five samples (samples A through E) were analysed and chemical material from inside containers and cover was sampled. The names are only "indicator information" until verified. The following are the results from each sample.

Sample “A” Ferric Chloride (Test result met expectations).

Test \#1 Result: Corrosive-pH reading not determined due to high staining of chemical on the test paper. Staining has masked any determination as to whether testing an acid base or neutral solution.

Test \#2a Result: Non-Flammable-The pH paper from Test \#1 was passed quickly through the flame for one second. There was no ignition and no colour change.

Test \#2b Result: Non-Flammable-The pH paper from Test \#1 was held in the flame for two seconds. There was no ignition, but the generated heat caused the release of acidic vapours that changed the $\mathrm{pH}$ paper to red. (The off gassing of the acidic vapours verifies the properties as being acidic. The colour of the reacted $\mathrm{pH}$ paper was consistent with the distinct colour of Sulphuric Acid vapours.

Test \#3 Result: Oxidizer-When white-coloured potassium iodide starch paper came in contact with the chemical, it turned dark yellow-brown and eventually over a four-hour time period turned yellow. On the high side of caution, any substance that causes any colour change from white in the starch paper should be considered an oxidizer.

\section{Sample "B" Sodium Hypochlorite (Solution)}

Test \#1 Result: Corrosive Base-The strip of $\mathrm{pH}$ paper was dipped into the sample. The $\mathrm{pH}$ paper immediately turned blue in colour for a few seconds and then the colour dissipated. Any bleaching on $\mathrm{pH}$ paper indicates the presence of an oxidizer and can be verified with starch paper in test \#3.

Test \#2a Result: Non-Flammable, No sign of ignition-The test paper used in test \#1 was passed quickly through the flame for one second. No signs of ignition or off gassing were observed.

Test \#2b Result: Non-Flammable, No sign of ignition-Test paper from Test \#1 was held in the flame for two seconds. There was no ignition and no colour change.

Test \#3 Result: Oxidizing Hazard-Potassium iodide starch paper was used to determine the oxidizer properties of the sample that could react with any combustible to create heat, off-gassing, fire or an explosion. 


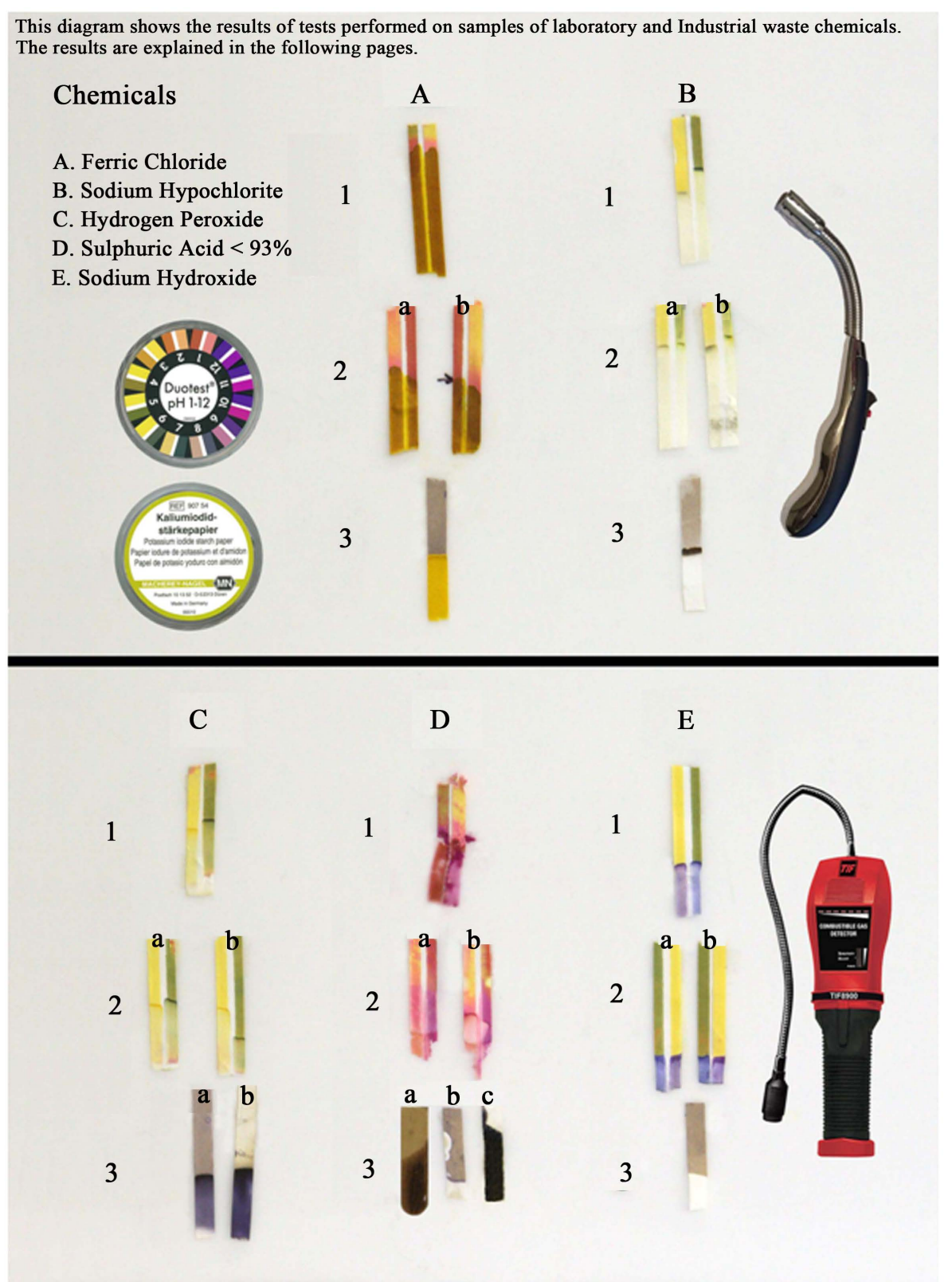

Figure 2. Results of tests performed on samples of laboratory and industrial waste chemicals.

(For any oxidizer review all site safety and compatibility of chemical storage.)

\section{Sample “C” Hydrogen Peroxide (household)}

Test \#1 Result: Corrosive-Test showed a pH of 4 - 5 with the colour slowly bleaching out. Any bleaching on $\mathrm{pH}$ paper indicates the presence of an oxidizer and can be verified with starch paper in test \#3.

Test \#2a Result: Non-Flammable-The test paper was held in the flame for one second and the heat immediately bleached the paper.

Test \#2b Result: Non-Flammable-The application of heat immediately bleached all of the colour from the $\mathrm{pH}$ paper.

Test \#3 Result: Oxidizer-The starch paper turned black when in contact with the solution therefore the substance is an oxidizer.

Note: It was also observed that the dry portion of the paper was getting darker as it was picking up airborne cross contamination from the sample within close proximity on the display board. This display was set up to 
have five liquid samples presented in tight format where trace amounts of chemical can be picked up by using the test papers as a sniffing tool.

\section{Sample "D" Sulphuric Acid Waste}

Test \#1 Result: Corrosive, Water and Heat Reactive-The test paper showed a pH of less than one. The Sulphuric acid is disintegrating the $\mathrm{pH}$ paper as it was being place on the display board. The reading can be very dark in colour. Therefore, to verify the acid property dissolve a few drops of sulphuric acid into $20 \mathrm{ml}$ of water and take another reading. Should you reach the $70 \%$ concentration, the test paper may disintegrate and dissolve into the water. The concentration of this waste sample is most likely $<93 \%$ and $>70 \%$ based on observation.

Test \#2a Result: Non-Flammable-A sample of the sulphuric acid was placed on a strip of $\mathrm{pH}$ paper for testing. The sample was passed through the flame quickly and the heat made the sulphuric acid slightly more reactive than it was at ambient temperature.

Test \#2b Result: Non-Flammable

A sample of the sulphuric acid on the end of a strip of $\mathrm{pH}$ paper was held in the flame for two seconds. The result was an immediate melting or decomposition of the test paper as the reaction from the heat of the lighter immediately demonstrated the heat reactive properties of sulphuric acid, which disintegrated the end of the test paper.

Test \#3 Result: Oxidizer-\#3a on Display Board had one drop of the waste Sulphuric Acid placed on a wooden stick and allowed to sit for 12 hours. The oxidizing properties of the acid charred the wood. This demonstrates an oxidizer can react with anything that burns to create heat, fire or an explosion.

\#3b is the original strip of starch paper used in the test and is included for illustration. The purpose of the five chemicals being displayed in close proximity to one another was to demonstrate how trace amounts of contamination being emitted from containers, processes or fume hoods can react and create unknown and unwanted reactions. The papers were left to dry over a 24-hour period.

Result: Sample D, \#3b had the oxidizing properties reversed through cross-contamination. The darker colour on the top portion is a result of the combined oxidation from the display, the white on the bottom end or tip and the white on the left side of the test paper were dark purple-black in colour when originally placed on the display.

\#3c was a fresh sample, which shows the original staining and colour of sample 3b. It can be speculated that the caustic vapour from sample E would have had the biggest nearby influence for the change! The sample has not been examined to determine if crystallization has occurred from the two corrosive atmospheres neutralizing themselves or masking the test results.

Sample "E" Sodium Hydroxide liquid (Caustic)

Test \#1 Result: Corrosive-Test paper showed a pH of 12 - 14.

Test \#2a Result: Non-Flammable-Paper did not ignite when held in the flame for one second.

Test \#2b Result: Non-Flammable-Paper did not ignite when held in the flame for two seconds.

Test \#3 Result: Non-Oxidizer-Initial test with starch paper confirms that the Sodium Hydroxide is a nonoxidizer. The top part of the highly sensitive starch paper-the dry part-indicates that there were trace amounts of oxidizing properties from other chemicals in close proximity. However, these did not change the reading for the portion of the paper dipped in caustic.

From the tests, we can conclude that anyone working with known and unknown chemicals in varying environments needs to understand the Golden Rules: Never assume, suit up for toxicity, work clean.

\subsection{Using $\mathrm{pH}$ Paper as a Search and Monitoring Tool}

This risk-management testing process to identify chemical properties in the field for personal safety, identification and classification provides responders with a process and tools to assist in dealing with all aspects of an event. Key factors are to identify and verify risks and hazards by using small to trace amount sized samples and swab tests. The tests can provide immediate and accurate information, support information and direction for an effective, timely response. Effective size-up, response, and remediation can be carried out in an orderly manner with less impact on the public, the environment and the workers themselves.

This approach for a safe response to incidents involving chemicals and hazardous wastes has been taught to personnel in universities, hospitals, police and fire hazmat teams, federal and provincial environmental agencies, nuclear plants and hydro generation stations, pulp and paper mills, mines, heavy industry and waste management. 
The high sensitivity of these test papers has been used to search out unknown sources of trace-chemical vapours and gasses, make chemical property determinations as well as for swab tests to identify and verify effective decontamination as they can be used wet or dry. When searching for gases or corrosive vapours, the DuoTest $\mathrm{pH}$ paper can be used effectively wet or dry, as some gases respond more readily to damp surfaces and others to dry paper.

\subsubsection{Corrosive Vapour Searching and Monitoring Tool}

Three-inch long pieces of pH paper will work best with personnel wearing gloves and carrying out other activities. The pH paper should be half-wet with distilled, household or stream water and half dry. The paper can be fastened to a face shield or clothing so that it is visible to the responder to allow monitoring of any colour change resulting from corrosive vapours. This will allow personnel to determine if they are properly protected to be in a corrosive atmosphere.

\subsubsection{Indoor Laboratory Corrosive Spills}

In spill conditions in laboratories, test paper should be half wet and half dry. It may pick up trace amounts of corrosive contamination on either the wet half of the strip or on the dry side, depending on the chemical. Testing dry powders inside containers can be done by obtaining a representative-sample on a glass rod and touching wet $\mathrm{pH}$ paper to the residue on the rod.

\subsubsection{Decontamination Verification}

In areas were trace amounts of contamination may be suspected or remain after spill cleanup, wet $\mathrm{pH}$ paper can be used to conduct intermittent swab tests in the immediate area to identify contamination or verify area is free from contamination. It should be noted that flammable hydrocarbons do not have a pH value, but will produce a reading on the DuoTest Paper of $\mathrm{pH} 4$ - 5. Therefore, this could indicate a mild acidic substance or flammable or combustible products. (Flash point estimates are covered in Test \#2.)

\section{Conclusions}

Safe response involves securing an area from unexpected interference, identifying safety and danger factors, assessing risks and hazards and determining the potential for an incident to escalate. However, the Quick Check Chemical Testing Process uses "hard-science”, the known physical properties of chemicals and chemical wastes, to determine reactions, scales of impact and critical timing. Normally it will take less than one minute to learn whether the chemicals are reducing agents, oxidizers, acids, bases, are heat or water reactive and what are their levels of flammability and corrosiveness.

In the hands of trained responders and other personnel, the Quick Check Chemical Testing Process, using a roll of pH paper, a roll of potassium iodide starch paper, a Bunsen burner or small portable propane torch and a portable gas sniffer, can provide accurate and objective information on the properties of known and unknown chemicals and chemical compounds. It removes the danger of speculation or guesswork and helps responders deal with the actual properties of what they are facing and choose appropriate personal protective equipment (PPE) for the situation.

In a cradle-to-grave chemical handling system, the Quick Check Chemical Testing Process is invaluable for overall workplace safety and safe handling, storage and disposal for all chemical waste streams on a site.

"Hard-science" provides factual information and identification of chemical properties that are necessary to handle, store, bulk and dispose of known and unknown chemicals and deal with hazardous wastes. The system used in our tests relies on this information for tests that can immediately identify and verify physical and chemical properties of known and unknown solids, liquids and airborne chemicals.

\section{References}

[1] Bretherick, L. (1990) Bretherick’s Handbook of Reactive Chemical Hazards. Butterworth International Edition, Butterworths.

[2] Lewis Sr., R.J. (2007) Hawley’s Condensed Chemical Dictionary. 15th Edition, John Wiley \& Sons, Inc., New York. http://dx.doi.org/10.1002/9780470114735 